\title{
Heritage Language Complexity Matters: The Editors' Introduction to the Special Issue
}

\author{
Oksana Laleko \\ Associate Professor, Linguistics Program and English Department, \\ State University of New York at New Paltz, USA \\ lalekoo@newpaltz.edu \\ Olesya Kisselev \\ Assistant Professor, Department of Bicultural Bilingual Studies, \\ University of Texas at San Antonio, USA \\ olesya.kisselev@utsa.edu
}

The story of heritage languages is often told as a story of grammatical simplification. Whether in the classroom or "in the wild" (Polinsky \& Kagan, 2007), heritage speakers have been the focus of investigations of processes and principles of linguistic change for over two decades. Nearly every subfield of language study has now addressed the heritage language phenomenon, entailing new interdisciplinary collaborations among scholars in formal and applied linguistics, sociolinguistics, language acquisition, contact linguistics, typology, classroom instruction, corpus research, and many other disciplines.

Notwithstanding the remarkable gains achieved across all of these areas, inherent within many underlying research traditions has been the practice of placing a heritage language system on one side of the scale, with an appropriately chosen baseline system balanced on the other side. Understood narrowly in such comparative terms, and setting aside the issue of proper calibration of the measuring mechanism employed, the question of what is interesting and novel about heritage languages is often asked through a deficit lens, bringing under the microscope those areas in which heritage languages fall short of displaying all of the properties and processes documented for earlier, pre-contact stages in their genesis.

Imagine what our conception of the grammatical structure of Modern English would be if all or most linguistic theorizing were carried out from a similarly-oriented "what's missing" perspective - say, in comparison to the structure of Old English or, more broadly, in relation to other Indo-European 
languages - without taking stock of the internal processes and developments that characterize the system under analysis on the synchronic level. Has Modern Literary Russian suffered an unfortunate fate in its gradual elimination of some of the inflectional exuberance of Old Church Slavonic? Reformulating the question in terms of tighter and more immediately observable diachronic boundaries: how much can we learn about the inner workings of the human mind, the intricacies of mechanisms involved in acquiring, storing, and accessing linguistic representations for comprehension and production, and the impact of the external, socio-historical correlates of linguistic variation and change, if our conception of heritage languages does not go far beyond reducing these varieties to imperfect replicas of their source grammars?

This special issue aims to enrich our understanding of complexity and the complex dynamics of heritage languages. In doing so, we bring together a diverse range of theoretical and applied perspectives representing generative, sociolinguistic, and typological linguistic traditions and intersect them with advances in heritage language instruction and assessment. This convergence of approaches allows us to identify (i.e., define and measure) complex phenomena across multiple domains of heritage grammars, account for the complex processes involved in their acquisition and use, and offer solutions for the preservation of heritage language complexity through targeted classroom intervention.

To the extent that it is possible to do justice to the rich and multidimensional nature of these matters within the scope of a dedicated issue, we organize the nine contributions of the present collection of articles into two volumes. Part 1: Theoretical Approaches to Heritage Language Complexity focuses on positioning and modeling the notion of linguistic complexity within the heritage language field, while Part 2: Applied Approaches to Heritage Language Complexity demonstrates how the various models and conceptualizations of complexity may be utilized and refined in applied contexts, including for instructional purposes. As the first collection of articles devoted to the topic of heritage language complexity, this special issue strives not only to reflect the current state of cross-disciplinary research on complexity (in)variance in heritage language settings, but also to advocate and lay the groundwork for future theoretical and empirical work that will expand our understanding of heritage language complexity in its many aspects and manifestations beyond the horizons of the presently available frameworks, methodologies, and data.

In the opening article, Oksana Laleko and Gregory Scontras offer a comprehensive scoping analysis of key issues pertaining to the multiple dimensions of linguistic complexity viewed through the lens of heritage language research. Taking stock of the last two decades of empirical work in heritage linguistics, 
they bring together insights from three broad strands of research on linguistic complexity, moving from attempts to arrive at objective or "absolute" metrics to assess the complexity of (sub)systems of grammar, to the search for sociodemographic correlates of variability in complexity, to its conceptualizations from the processing and acquisitional standpoints that center on the language user (i.e., "relative" complexity). At each junction, the authors emphasize how our current understanding of heritage language systems and of the processes involved in their formation and use may both inform and be informed by the existing conceptualizations and models of linguistic complexity.

Working within the generative framework, Terje Lohndal and Michael Putnam propose a heuristic that decomposes the notion of grammatical complexity into three smaller formal components: syntactic features, their hierarchical organization, and the mapping between syntactic features and their exponents. Assuming a distributed model of the lexicon, in which linguistic information is integrated across several modules in the architecture of grammar, the authors conceptualize heritage language complexity as resulting from the geometry of features, projections, and computational operations, arguing that "any change in the feature system of a language is not simply a lexical property of a grammar, but rather is properly understood as a structural one" (p. 22). Drawing on their own analysis of the grammatical gender system in American Norwegian and other published data, Lohndal and Putnam offer a featural typology of complexity changes in heritage grammar formation. The proposed heuristic accounts for instances of decreasing complexity, understood here as a reduction in the number of features or projections, and instances of increasing complexity, conceptualized in terms of mismatches between features and their formal exponents (cf. Miestamo, 2008).

Shifting the focus from representational approaches to heritage language complexity to its computational, processing-based effects, Silvina Montrul surveys recent studies on gender agreement and differential object marking to demonstrate that differences between baseline and heritage grammars do not necessarily lie at the level of syntactic representations, but may instead be manifested in the speed of lexical access and online feature reassembly. In this approach, termed the Differential Lexical and Syntactic Activation Hypothesis, heritage speakers at intermediate and high levels of proficiency may develop the same linguistic representations as baseline speakers, but display less efficiency with lexical access and the construction of syntactic projections. Building on models that account for difficulties with functional morphology in second language acquisition, Montrul argues that heritage language acquirers may face unique computational pressures in producing and comprehending the heritage language, stemming from the diminished frequency and 
consistency of their linguistic exposure, lower degrees of language entrenchment, and cross-language competition. In this scheme, some of the formal features of the heritage language may be arranged similarly to the baseline, but remain weakly activated, deactivated, or dormant, resulting in variable assignment of morphophonological content. Crucially, then, an apparent simplification of surface forms is not necessarily a marker of restructuring of the underlying linguistic structures, and the inherent variability observed across heritage language systems at different proficiency levels is best accounted for when representational and computational possibilities are considered jointly.

Looking at complexity through a sociolinguistic lens, Naomi Nagy and Timothy Gadanidis undertake a comparative analysis of heritage and homeland Cantonese, with an eye to the degree of complexity instantiated in both varieties across two distinct dimensions: in the use of surface forms and in the decision-making process involved in their choice. The authors draw on a variationist analysis of prodrop and classifiers, two phenomena that require the acquisition of linguistic structures along with probabilistic information about their appropriateness (a Variability Matrix), to demonstrate that heritage and homeland speakers utilize equally complex processes in selecting the relevant forms. The variationist perspective employed in this study offers a unique practical conceptualization of complexity in terms of the number of significant factors deterministically involved in the distribution of the relevant structures: "If more factors significantly affect (or correlate to) the variation in Variety A than Variety B, we may say that Variety A is more complex" (p. 3). In addition to the proposed quantitative measure of complexity, Nagy and Gadanidis make a methodological contribution to the field of heritage language studies by demonstrating the advantages of the statistical mechanism of bootstrapping, which allows for reliable comparisons across language varieties based on samples of unequal size - a common issue in work on heritage and endangered languages.

Anchoring their conceptualization of complexity to sound systems, Robert Klosinski and Andrew Hoffman examine the comparative complexity of phonological structures in two varieties of heritage Bernese Swiss German, spoken in Argentina (Misiones) and the United States (Ohio), with a focus on its two salient characteristics, /l/-vocalization and /nd/-velarization. Following a comprehensive review of existing models and conceptualizations of complexity in language systems, the authors propose to operationalize phonological complexity as a two-dimensional construct, drawing a distinction between complexity of structure and complexity of process. The results of the study provide a strong argument for considering both dimensions in tandem. While /1/-vocalization 
appears to be intact in both communities, the loss of /nd/-velarization documented in Misiones serves to represent a decrease in processual complexity in this variety of heritage Bernese, manifested as a greater degree of transparency between underlying forms and their realizations. However, the surface outcome of this change - the use of coda consonant clusters by heritage speakers - is a case of increased structural complexity. It is notable that the Misiones speakers of Bernese opted for producing complex clusters despite pressures from the relatively simpler phonological structure of Spanish, a finding that calls into question previous accounts of heritage language complexity change in terms of convergence towards the simpler structure of those present in the bilinguals' competing languages.

Julio Torres turns the discussion to the heritage language classroom as he reflects on the role of complexity in the emerging field of instructed heritage language acquisition (IHLA). Focusing on complexity conceptualized through user-based parameters such as transparency, saliency, and communicative value (or "cognitive complexity" in the author's terms), Torres summarizes the lessons learned in the field of instructed second language acquisition and considers them in relation to IHLA. He reassesses an empirical study on the development of Spanish gender assignment and agreement in writing (Pérez-Núñez, 2018) through a complexity lens, presenting a potent argument in favor of integrating the factor of morphosyntactic complexity of target forms into the study of the traditional factors assumed to impact IHLA, including previous experience with language, individual differences, and type of instruction. Looking ahead, he advocates for a more nuanced understanding of the dynamics among these variables as a foundation for the development of evidence-based guidelines and principles for heritage language instruction.

In a related vein, Melissa Bowles addresses the issue of pedagogical approaches in the heritage language classroom by examining learning gains in Spanish heritage language learners subjected to either explicit or implicit "focus on form" instruction. Concentrating on the role of rule presentation, the study compares language gains in heritage learners of Spanish in implicit and explicit conditions as the learners are presented with instruction on indicative and subjunctive constructions in adverbial clauses of time. Analysis of the comparisons on the pre-tests and the immediate and delayed post-tests demonstrates that both types of instruction lead to lasting learning gains, highlighting the complex interplay between the learners' initial knowledge of the structure and the type of instruction received. Thus, learners with little or no initial knowledge of the relevant structure appear to benefit from rule presentation, whereas learners with greater initial knowledge do not. These 
empirical results support the general argument of Torres' paper and provide vital evidence of the complex dynamics of heritage language acquisition in classroom settings.

Employing computational methods utilized in second language complexity research, Olesya Kisselev, Mihail Kopotev, and Aleksandr Klimov undertake a corpus-based study of complexity in heritage language writing, focusing on heritage learners of Russian at different proficiency levels. Having compared essays grouped into various proficiency levels along 12 automatically extracted measures of syntactic complexity (which included average sentence length, average amounts of coordinate clauses and subordinate clauses, and measures of phrasal depth, among others), the authors found that differences in the counts of the majority of the automatically extracted measures - nine of the 12 selected - reached statistical significance. Specifically, such measures as sentence length, phrasal depth and specific types of subordinate clauses (although not the amalgam measure of subordination) appeared to reliably differentiate proficiency levels of heritage speakers of Russian. The results of the analysis not only suggest that the indices of syntactic complexity frequently used in the study of second language development may serve as correlates of the proficiency levels of heritage speakers, but also demonstrate first-hand the importance of carefully considering the variability in attained heritage language proficiency as a predictor of linguistic complexity across syntactic structures.

John Lipski takes readers into the field, to Misiones province, Argentina, to examine the sensitivity of Spanish-dominant Brazilian Portuguese heritage speakers to putative grammatical constraints on intrasentential code-switching in an environment where such knowledge could not have been derived from these speakers' linguistic input. Participants were offered a series of interactive tasks, from two-alternative forced-choice tests to an eye-tracking experiment, to further the issue of acceptability beyond simple ratings and to provide an insight into the online processing of code-switching by heritage language speakers. Viewed as relative measures of attainment in heritage bilingual grammars, these tasks confirmed the disfavored status of switches after subject pronouns and fronted interrogative words, as well as between modals and infinitives. Cautioning against conceptualizations of heritage languages in terms of acquisitional deficits, Lipski interprets these results as an argument in favor of heritage speakers demonstrating high levels of sensitivity to complex grammatical constraints on code-switching, a marker of the robustness and cohesiveness of the acquired bilingual system and its elaborate semantic organization.

A fitting envoi to the collection, John McWhorter's book review of the Oxford Handbook of Language Attrition, edited by Monika Schmid and Barbara 
Köpke, provides an astute perspective on the key theoretical problems and empirical questions positioned at the intersection between the fields of attrition and heritage language studies. In drawing parallels and highlighting differences between these adjacent but independently evolving areas of inquiry, McWhorter offers an engaging critical commentary of the recent advances across this wide research spectrum, taking up the issue of linguistic complexity as its central unifying motif. Starting with the assumption that "[a] Il languages vastly overdo what is necessary to rich and nuanced communication" (p. 8), McWhorter strongly cautions against mischaracterizations of complexity of structure in terms of complexity of thought. He urges scholars in both fields to work more collaboratively in striving not only to heighten the level of precision in constructing scientific models, but also to expand their terminological resources when communicating ideas across disciplinary boundaries and beyond academic settings.

As these brief summaries illustrate, the rich and multifaceted topic of heritage language complexity has steadily found its way into the field and will undoubtedly impact the trajectory of heritage language research for years to come. We are also reminded, as we embark on the exciting work ahead, that we are fortunate to be able do so while standing on the shoulders of giants. A great many questions raised in the contributions to this special issue are rooted in long-standing debates in the vast literature on complexity, bridging insights from historical and typological research, creole studies and language contact, acquisition, sociolinguistics, and computational approaches. Moving forward, we envision rich opportunities for our young field to partake fruitfully in the multidisciplinary endeavor that will bring us closer to understanding complexity as a fundamental property of human language.

\section{Notes on Contributors}

Oksana Laleko is an associate professor of Linguistics and Director of the Linguistics Program at the State University of New York (SUNY) at New Paltz. Her scholarly contributions include articles and book chapters on linguistic and cognitive aspects of bilingualism. Her recent work focuses on the syntaxdiscourse interface in heritage language and L2 acquisition.

Olesya Kisselev is an assistant professor in the Department of BiculturalBilingual Studies, College of Education and Human Development at The University of Texas at San Antonio. Her primary research interests include corpus linguistics and discourse analysis, especially as they apply to the study 
of various aspects of second language and heritage language acquisition. Her scholarly contributions include research papers and book chapters on linguistic aspects of heritage learner language as well as on heritage language pedagogy.

\section{References}

Miestamo, M. (2008). Grammatical complexity in a cross-linguistic perspective. In M. Miestamo, K. Sinnemäki, \& F. Karlsson (Eds.), Language complexity: Typology, contact, change (pp. 23-41). John Benjamins.

Pérez-Núñez, A. (2018). The acquisition of Spanish gender marking in the writing of heritage and second language learners. Heritage Language Journal, 15(2), 242-267. https://doi.org/10.46538/hlj.15.2.4.

Polinsky, M., \& Kagan, O. (2007). Heritage languages: In the 'wild' and in the classroom. Language and Linguistics Compass, 1(5), 368-95. https://doi.org/10.1111/j.1749 $-818 X .2007 .00022 . x$. 